\title{
Gastrointestinal symptoms in travellers
}

\author{
Authors: Alastair C McGregor ${ }^{A}$ and Stephen G Wright ${ }^{B}$
}

Diarrhoea is probably the single most common medical complaint in returning travellers. The most common pathogens are entero-toxigenic Escherichia coli, Shigella, Salmonella and Campylobacter. Viruses, toxigenic Arcobacter and Bacteroides fragilis, as well as parasites such as Cryptosporidium sp, are increasingly recognised but are not tested for in most diagnostic laboratories. Blood in stools is a sign of invasive disease and should trigger exclusion of invasive amoebic disease. The use of empiric antibiotics may shorten illness but is complicated by the diversity of bacterial causes and emerging resistance.

\section{Diarrhoea is common in travellers}

\section{Travel broadens the mind and loosens the bowels}

Diarrhoea, defined as the passage of watery or loose stool at least three times in 24 hours, ${ }^{1}$ is common in travellers. An estimated 40,000 visitors to low- and middle-income countries develop diarrhoea every day. ${ }^{2}$ Generally, diarrhoeal diseases are linked to poor hygiene. In the UK, we take clean water and sanitation, both personal and public, for granted. In the developing world, however, standards of hygiene are often poor and existing sanitation provisions may be further compromised by natural disasters and population displacement in times of famine or conflict.

\section{Differences between small and large bowel diarrhoea}

The cause of diarrhoea can often be localised to the small or large bowel on clinical grounds. Small bowel disease results in large-volume stools, with pain a relatively uncommon symptom. Pathogens involved include viruses (norovirus, rotavirus and astrovirus), toxigenic bacteria such as enterotoxigenic Escherichia coli (ETEC), Vibrio cholerae and Clostridium perfringens, and protozoan parasites (Giardia lamblia, Cryptosporidium and Cyclospora spp). Bacterial and viral enterotoxins increase efflux of chloride, sodium and water in the small bowel to cause net fluid secretion.

Authors: ${ }^{A}$ SpR, Hospital for Tropical Diseases, London, UK, and Imported Fever Service, Rare and Imported Pathogens Laboratory, Public Health England, Porton Down, UK; ${ }^{B}$ consultant physician, Hospital for Tropical Diseases, London, UK, and consultant physician, King Edward VII Hospital, London, UK
The pathogenesis of diarrhoea caused by Giardia and Cryptosporidium is incompletely understood. Giardia is able to disrupt the epithelial brush border and intercellular adhesion, while increasing enterocyte apoptosis, leading to enterocyte dysfunction. ${ }^{3}$ Luminal digestion also seems to be impaired in giardiasis. These changes result in malabsorption and diarrhoea. ${ }^{4}$ Cryptosporidia inhabit an intracellular but extracytoplasmic compartment within enterocytes. Infection leads to effacement of the microvilli and alterations of fluid and electrolyte absorption through poorly characterised mechanisms.

Most of the fluid secreted into the small bowel is reabsorbed and over 24 hours only $1-1.51$ of fluid enters the colon. When colonic absorption is impaired, the volume of stool produced is therefore much less than in small bowel disease. Cramping abdominal pain due to colonic wall contractions is prominent in large bowel infection and ulceration of inflamed colonic mucosa may lead to visible bloodstaining of stools. Urgency of defaecation with tenesmus is characteristic of rectal inflammation.

\section{Time course of diarrhoea}

The length of a diarrhoeal illness may provide some clues to the cause. Watery diarrhoea of acute onset and short duration is caused by enterotoxigenic bacteria or viral infections. Some bacteria, such as Staphylococcus aureus and Bacillus cereus are capable of producing enterotoxins ex vivo and ingestion of foods contaminated with preformed toxin can cause vomiting and diarrhoea with a rapid and sudden onset (4-6 hours). Giardiasis, cryptosporidiosis and cyclosporiasis can also cause acute watery diarrhoeal illnesses but they tend to run a more prolonged course.

\section{Presence of blood in the stools}

Invasive colonic infection is often associated with systemic illness characterised by abdominal pain and fever. Luminal bleeding from inflamed mucosa is typical in invasive infection but is not always clinically apparent. The term dysentery refers to haemorrhagic diarrhoea with systemic upset. The most common bacterial causes are species of Shigella, Salmonella and Campylobacter, as well as enteroinvasive and enterohaemorrhagic E coli. ${ }^{5}$

Infection with the protozoan parasite Entamoeba histolytica can cause a colitis that is clinically indistinguishable from ulcerative colitis. The epidemiology of this organism is better 
understood now that its encysted form can be distinguished from the morphologically identical but non-pathogenic Entamoeba dispar and Entamoeba moshkovskii using molecular tests. Epidemiological studies in Vietnam have revealed that only about $10 \%$ of individuals excreting cysts of E histolytica/ dispar actually have infection with E histolytica. Amoebiasis may be missed as the cause of dysentery if fresh samples from stool or rectal scrapes are not examined promptly for amoebic trophozoites. Exclusion of amoebiasis is crucial prior to immunosuppressive therapy in cases of presumed non-specific inflammatory bowel disease.

\section{Infectious causes of diarrhoea in travellers}

\section{Viral pathogens}

Norovirus is probably the single most common viral pathogen associated with diarrhoea in travellers. ${ }^{6}$ Polymerase chain reaction (PCR) testing is the usual means of diagnosis but this technique is not available in communities in the tropics. Rotavirus is less common in adults and its worldwide incidence is likely to fall further following widespread introduction of childhood vaccination.

\section{Bacterial infections and Clostridium difficile}

Infection with enterotoxigenic $E$ coli and $V$ cholerae causes painless diarrhoea with large-volume watery stools. The main symptoms and physical signs relate to fluid depletion: thirst, dizziness and postural hypotension without systemic upset. Invasive bacterial organisms cause colicky abdominal pain, systemic upset and often bloodstained stools.

Most laboratory protocols allow identification of Campylobacter, Shigella, Salmonella spp and E coli O157. Testing for ETEC and newly identified pathogens, such as enterotoxigenic Bacteroides fragilis and Arcobacter spp, is not available in routine diagnostic laboratories. The diagnostic yield of PCR-based assays is much greater than traditional culture-based methods. ${ }^{7}$ However, the specificity of these tests is impossible to ascertain because of the lack of a reliable gold standard.

\section{Key points}

Diarrhoea is a major complaint in travellers

Small bowel disease is characterised by high-volume, watery stools, whereas colonic infection tends to cause loose stool, often associated with blood staining and systemic illness

Non-infectious causes of diarrhoea should be considered in individuals with persistent or severe symptoms

Exclusion of amoebiasis is important in travellers with bloody diarrhoea, particularly if immunosuppression is being considered

KEYWORDS: Diarrhoea, gastroenteritis, travel, travellers, imported
Despite the ready availability of antibiotics without prescription in the tropics, the frequency of $C$ difficile-toxin associated diarrhoea in travellers has been little studied. It is likely to be relatively common and should be considered in all patients with diarrhoea and a history of antibiotic use.

\section{Protozoa}

Giardia intestinalis, Cryptosporidium spp and E histolytica are the common protozoal causes of diarrhoea, with Cyclospora increasingly recognised. Giardia is a common cause of acute, persisting and chronic diarrhoea in travellers. Foul-smelling flatus and stools, lethargy and abdominal distension are common symptoms in patients with more severe infections. Cryptosporidiosis results in a non-specific diarrhoeal illness with similar clinical features to giardiasis. ${ }^{8}$ In immunocompetent individuals, self-limiting infection is the rule while, in the presence of immunodeficiency or malnutrition, persisting symptomatic infection is common.

Only a small number of individuals who excrete cysts of E histolytica are symptomatic. In endemic populations, it is estimated that about $1 \%$ of infected individuals develop invasive disease and the estimated half-life of an infection is 13 months. ${ }^{9-10}$ The rectum and caecum are sites of predilection for mucosal invasion but any length of colon can be affected with increasing severity of symptoms with greater extent of colitis. Abdominal tenderness varies according to the severity of disease. Like all inflammatory diseases of the colon, whether inflammatory or infective, toxic dilatation and perforation can occur.

\section{Helminths}

Gut nematodes rarely cause diarrhoea. Strongyloidasis is a fairly common cause of abdominal upset, but frank diarrhoea appears to be uncommon. Acute heavy hookworm infection can cause diarrhoea in travellers. ${ }^{11}$ Schistosoma mansoni is occasionally associated with macroscopic gastrointestinal (GI) bleeding and diarrhoea.

\section{Tropical sprue}

Tropical Sprue, a post-infective malabsorption syndrome, was described in South Asians, British soldiers in Malaysia and in overland travellers to Asia (but not to Africa). Malabsorption and jejunal mucosal abnormalities were described. In contrast to coeliac disease, vitamin B12 malabsorption was an unusual finding. ${ }^{14}$ Cases are presently described by Indian clinicians but it is rare in travellers. Colonic cancer should always be considered in individuals with bloody diarrhoea, regardless of the travel history.

\section{Non-infective causes of diarrhoea}

Non-infectious causes of diarrhoea should be considered in travellers with prolonged or severe GI symptoms. The most common cause of persistent diarrhoea is a post-infectious enteropathy, which occurs in as many as $10 \%$ of cases of travelassociated diarrhoea. ${ }^{12}$ Gastrointestinal conditions that are not primarily associated with travel, such as coeliac disease and inflammatory bowel disease can, of course, present for the 
first time in returned travellers. Underlying non-infectious GI pathology may become overt in the context of acute infection but the suggestion that ulcerative colitis can be triggered by infection is subject to detection bias and remains unproven. ${ }^{13}$

\section{Empirical treatment}

About $50 \%$ of cases of acute travel-associated diarrhoea resolve within 48 hours. ${ }^{6}$ However, a meta-analysis of six studies showed that prompt antibiotic administration in non-travellers increased the likelihood of resolution of symptoms within 72 hours almost six fold. ${ }^{15}$ Antibiotics may be less effective in travellers because of the different aetiology of diarrhoeal illness in this population and because of increasing rates of microbial resistance, particularly in Asia. Parasitic infections tend to cause longer illness and empiric treatment for giardiasis is worth considering in individuals with persistent symptoms. The absence of faecal white cells, fever or a raised C-reactive protein favour a parasitic cause of diarrhoea in travellers. ${ }^{16}$

\section{References}

1 Steffen R. Epidemiology of traveler's diarrhea. Clin Infect Dis 2005;41:S536-40.

2 Guerrant RL, Van Gilder T, Steiner TS et al. Practice guidelines for the management of infectious diarrhea. Clin Infect Dis 2001;32:331.

3 Hill DR, Nash TE. Giardia lamblia. In: Mandell GE, Bennett JE and Dolin R (eds), Mandell, Douglas, and Bennett's principles and practice of infectious disease, 7th edn, vol 2. Philadelphia: Churchill Livingstone, 2012:3527-9.

4 Buret AG. Pathophysiology of enteric infections with Giardia duodenalis. Parasite 2008;15:261-5.

5 Peltola H, Gorbach SL. Travelers' diarrhea - epidemiology and clinical aspects. In: DuPont HL, Steffen R (eds), Textbook of travel medicine and health. Hamilton, ON: Blackwell Science, 1997.
6 Ajami N, Koo H, Darkoh C et al. Characterization of norovirusassociated traveler's diarrhea. Clin Infect Dis 2010;51:123-30.

7 de Boer RF, Ott A, Kesztyus B, Kooistra-Smid AM. Improved detection of five major gastrointestinal pathogens by use of a molecular screening approach. J Clin Microbiol 2010;48:4140-6.

8 Weitzel T, Wichmann O, Mühlberger N et al. Epidemiological and clinical features of travel-associated cryptosporidiosis. Clin Microbiol Infect 2006;12:921-4.

9 Gathiram V, Jackson T. Frequency distribution of Entamoeba histolytica zymodemes in a rural South African population. Lancet 1985; 325:719-21.

10 Blessmann J, Ali IK, Nu PA et al. Longitudinal study of intestinal Entamoeba histolytica infections in asymptomatic adult carriers. J Clin Microbiol 2003;41:4745-50.

11 Lawn S, Grant A and Wright SG. Case reports: acute hookworm infection: an unusual cause of profuse watery diarrhoea in returned travellers. Trans R Soc Trop Med Hyg 2003;97:414-5.

12 Okhuysen PC, Jiang ZD, Carlin L et al. Post-diarrhea chronic intestinal symptoms and irritable bowel syndrome in North American travelers to Mexico. Am J Gastroenterol 2004;99:1774-8.

13 Mann EA, Saeed SA. Gastrointestinal infection as a trigger for inflammatory bowel disease. Curr Opin Gastroenterol 2012;28:24-9.

14 Langenberg MC, Wismans PJ, van Genderen PJ. Distinguishing tropical sprue from celiac disease in returning travellers with chronic diarrhoea: A diagnostic challenge? Travel Med Infect Dis 2014;12:401-5.

15 de Bruyn G, Hahn S, Borwick A. Antibiotic treatment for travellers' diarrhoea. Cochrane Database Syst Rev 2000:3;CD002242.

16 McGregor AC, Whitty CJ, Wright SG. Geographic, symptomatic and laboratory predictors of parasitic and bacterial causes of diarrhoea in travellers. Trans R Soc Trop Med Hyg 2012;106:549-53.

Address for correspondence: Dr S Wright, King Edward VII Hospital, London W1G 6QA, UK.

Email: stephenwright1@doctors.net.uk 